\title{
Author Correction: Phonological network fluency identifies phonological restructuring through mental search
}

\author{
Karl David Neergaard 10 , Jin Luo \& Chu-Ren Huang \\ Correction to: Scientific Reports https://doi.org/10.1038/s41598-019-52433-w, published online 05 November \\ 2019
}

The HTML and PDF versions of this Article contained a typographical error in the spelling of the author Chu-Ren Huang, which was incorrectly given as Chu -Ren Huang.

In addition, the original version of this Article contained errors in the Abstract.

"Post exclusion, 95 native-language Mandarin speakers produced as many items that differed by a single lexical tone as possible within one minute.."

now reads:

"Post exclusion, 95 native-language Mandarin speakers produced as many items that differed by a single segment or lexical tone as possible within one minute..

Lastly, the original article contains errors in the Methods section under the subheading 'Fluency'.

"We introduce 'weighted edit' (WE), shown in Fig. 1b. WE states that a given word's value to the final fluency score decreases as edit distance between itself and the auditory stimuli increases. Fluency weightings differed according to the number of units for each of the stimuli and production, such that weightings were the proportion of the number of edit distances possible prior to two items sharing no similarity. For instance, for four-unit stimuli that received five-unit responses, edit 1 garnered 1 point (e.g., wai4/uai ${ }^{5151} / \rightarrow$ shuai $4 /$ suar $^{5151} /$ ), edit $2=0.75$ (e.g., wai4/uai ${ }^{5151} / \rightarrow$ shuan $4 /$ șuan $^{5151} /$ ), edit $3=0.50$ (e.g., wai4/uai ${ }^{5151} / \rightarrow$ xiang4/ (ian $^{5151} /$ ), edit $4=0.25$ (e.g., wai4/ uai $^{5151} / \rightarrow$ xiang $3 /$ cian $^{214} /$ ), and edit $5=0$ (e.g., wai $4 /$ uar $^{5151} / \rightarrow$ xiong3/(6ion ${ }^{214} /$ ). Similarly, for three-unit stimuli that received two-unit responses, edit $1=1\left(\right.$ e.g., du2 $\left./ \mathrm{tu}^{3535} / \rightarrow \mathrm{wu} 2 / \mathrm{u}^{3535} /\right)$, edit $2=0.50\left(\right.$ e.g., tu2 $/ \mathrm{tu}^{3535} / \rightarrow \mathrm{wu} 4 / \mathrm{u}^{5151} /$ ), edit $3=0$ (e.g., tu $\left.2 / \mathrm{tu}^{3535} / \rightarrow \mathrm{yi} 4 / \mathrm{i}^{5151} /\right)$, edits 4 and 5 also received 0 points due to not being possible combinations."

now reads:

"We introduce 'weighted edit' (WE), shown in Fig. 1b. WE states that a given word's value to the final fluency score decreases as edit distance between itself and the auditory stimuli increases. Fluency weightings differed according to the number of units for each of the stimuli and production, such that weightings were the proportion of the number of edit distances possible prior to two items sharing no similarity. For instance, for four-unit stimuli that received five-unit responses, edit 1 garnered 1 point (e.g., wai4/uai ${ }^{51} / \rightarrow$ shuai $4 /$ sua $^{51} /$ ), edit $2=0.75$ (e.g., wai4 $/$ uar $^{51} / \rightarrow$ shuan $4 / \operatorname{suan}^{51} /$ ), edit $3=0.50$ (e.g., wai $4 /$ ual $^{51} / \rightarrow$ xiang $4 / \operatorname{cian}^{51} /$ ), edit $4=0.25$ (e.g., wai $4 /$ uai $^{51} / \rightarrow$ xiang $3 / \operatorname{cian}^{214} /$ ), and edit $5=0$ (e.g., wai $4 /$ uar $^{51} / \rightarrow$ xiong $3 /$ cion $^{214} /$ ). Similarly, for three-unit stimuli that received two-unit responses, edit $1=1$ (e.g., du2/tu $\mathrm{tu}^{35} / \rightarrow$ wu $2 / \mathrm{u}^{35} /$ ), edit $2=0.50\left(\right.$ e.g., tu $2 / \mathrm{tu}^{35} / \rightarrow$ wu $4 / \mathrm{u}^{51} /$ ), edit $3=0$ (e.g., tu2 $\left./ \mathrm{tu}^{35} / \rightarrow \mathrm{yi} 4 / \mathrm{i}^{51} /\right)$, edits 4 and 5 also received 0 points due to not being possible combinations."

These errors have now been corrected in the PDF and HTML versions of the published Article. 
(c) (i) Open Access This article is licensed under a Creative Commons Attribution 4.0 International License, which permits use, sharing, adaptation, distribution and reproduction in any medium or format, as long as you give appropriate credit to the original author(s) and the source, provide a link to the Creative Commons license, and indicate if changes were made. The images or other third party material in this article are included in the article's Creative Commons license, unless indicated otherwise in a credit line to the material. If material is not included in the article's Creative Commons license and your intended use is not permitted by statutory regulation or exceeds the permitted use, you will need to obtain permission directly from the copyright holder. To view a copy of this license, visit http://creativecommons.org/licenses/by/4.0/.

(C) The Author(s) 2020 